Projets

de paysage

\section{Projets de paysage}

Revue scientifique sur la conception et l'aménagement de l'espace

16 | 2017

Arbres et paysages

\title{
Une approche multidisciplinaire de la fabrique des paysages dans la longue durée dans les forêts de Blois, Russy, Boulogne et Chambord (Loir-et-Cher)
}

A Multidisciplinary Approach to the Long-Term Production of Landscapes in the Forests of Blois, Russy, Boulogne and Chambord (Loir-et-Cher)

\section{Aude Crozet, Clément Laplaige et Xavier Rodier}

\section{OpenEdition}

\section{Journals}

Édition électronique

URL : http://journals.openedition.org/paysage/5487

DOI : 10.4000/paysage.5487

ISSN : 1969-6124

Éditeur :

École nationale supérieure du paysage de Versailles-Marseille, Institut national des sciences appliquées Centre Val de Loire - École de la nature et du paysage, École nationale supérieure d'architecture et de paysage de Bordeaux, École nationale supérieure d'architecture et de paysage de Lille, Agrocampus Angers

Référence électronique

Aude Crozet, Clément Laplaige et Xavier Rodier, « Une approche multidisciplinaire de la fabrique des paysages dans la longue durée dans les forêts de Blois, Russy, Boulogne et Chambord (Loir-et-Cher) », Projets de paysage [En ligne], 16 | 2017, mis en ligne le 06 juillet 2017, consulté le 30 avril 2020. URL http://journals.openedition.org/paysage/5487 ; DOI : https://doi.org/10.4000/paysage.5487

Ce document a été généré automatiquement le 30 avril 2020.

Projets de paysage 


\title{
Une approche multidisciplinaire de la fabrique des paysages dans la longue durée dans les forêts de Blois, Russy, Boulogne et Chambord (Loir-et-Cher)
}

\author{
A Multidisciplinary Approach to the Long-Term Production of Landscapes in the \\ Forests of Blois, Russy, Boulogne and Chambord (Loir-et-Cher)
}

Aude Crozet, Clément Laplaige et Xavier Rodier

1 Le paysage est défini par l'historien Gérard Chouquer (2000, p. 189) comme « l'ensemble des formes et des modelés visibles à la surface du sol ». En archéologie, il est la somme d'une accumulation de phénomènes et de pratiques sociales qui interagissent avec des composantes naturelles, s'inscrivent et perdurent (ou non) dans le temps et dans l'espace. L'archéologie permet d'aborder cette construction du paysage dans le temps long selon ses aspects spontanés ou programmés. La forêt est un milieu de conservation efficace des vestiges archéologiques, car l'érosion y est moins forte qu'en milieu ouvert, elle constitue par là un environnement privilégié pour l'étude du paysage.

Le programme SOLiDAR ${ }^{1}$ a pour objectif d'étudier de façon diachronique la fabrique du paysage sur un espace de $270 \mathrm{~km}^{2}$ comprenant le domaine national de Chambord ainsi que les forêts domaniales de Boulogne, Russy et Blois, soit un espace forestier de près de 25000 ha (figure 1). Outre les sources habituellement utilisées par les archéologues (fouilles et prospections pédestres, archives, études géomorphologiques et écologiques), des techniques de prospection de pointe (géophysique, télédétection) sont également utilisées. La zone étudiée était jusque-là connue grâce à de nombreuses campagnes de prospection pédestres menées bénévolement par Louis Magiorani depuis 15 ans. Une campagne complémentaire de télédétection LiDAR (Light Detection And Ranging) a été menée durant l'hiver 2015. 
Figure 1. Emprise de la zone d'étude

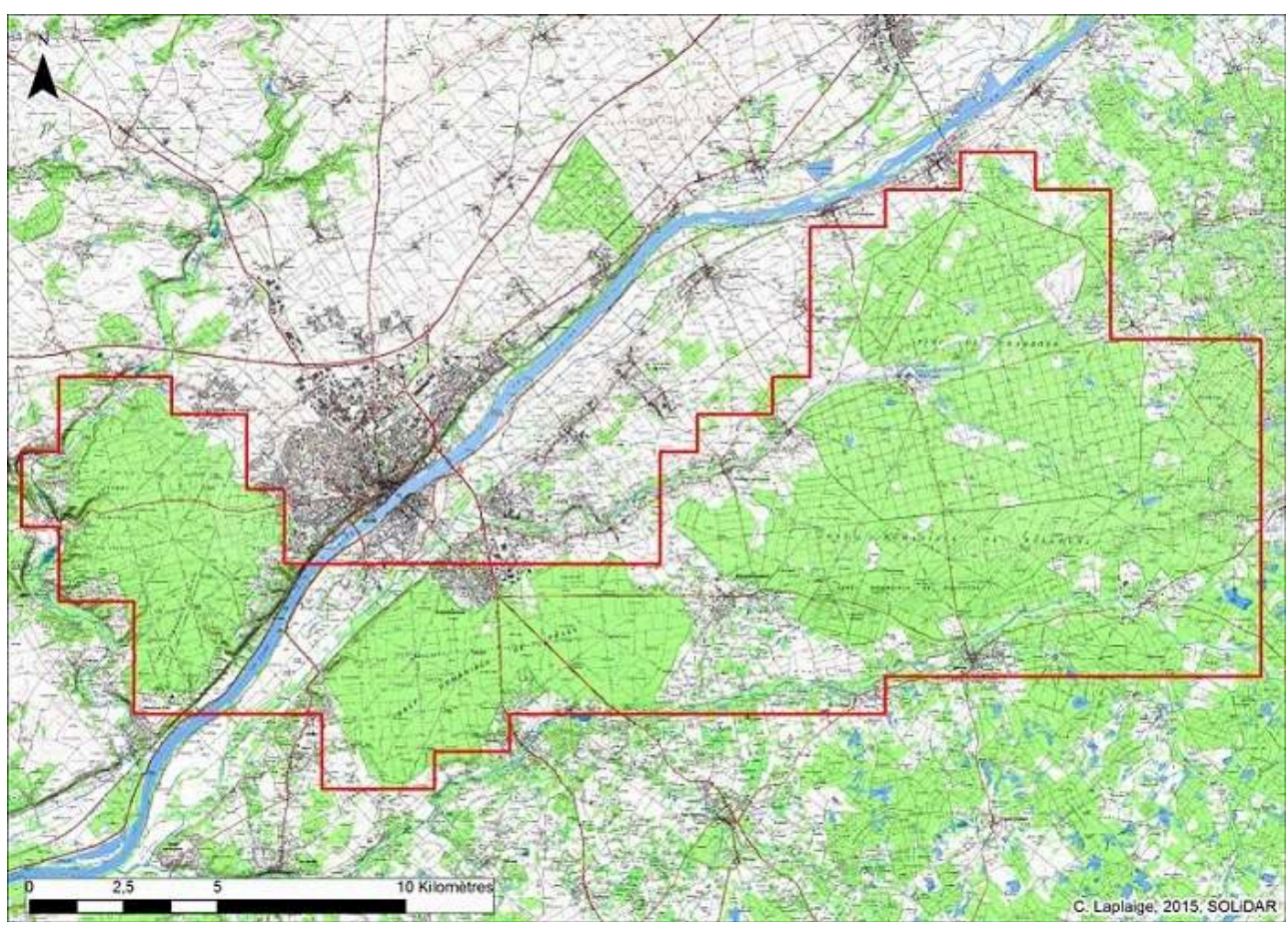

Source : Clément Laplaige, 2015, SOLiDAR.

3 Le concept de «fabrique » utilisé ici est dérivé du terme de fabrique urbaine développé par Henri Galinié. Il désigne un processus «par lequel l'interaction entre société urbaine et ville, dans sa réalité matérielle, espaces et territoires, produit un urbain spécifique en perpétuelle transformation »(Galinié, 2000, p. 126)», dans notre cas, l'interaction entre société rurale et espace forestier. Autrement dit, nous nous proposons d'aborder ici, via la méthode archéologique, les phénomènes historiques d'origine sociale qui ont contribué à façonner le paysage forestier dans cette partie du bassin de la Loire dans le temps long.

Après une présentation des sources utilisées, nous proposons une première reconstitution de l'histoire de l'exploitation des forêts concernées à travers les sources écrites et abordons, dans une troisième partie, les premiers résultats obtenus et les apports du LiDAR à la recherche archéologique en contexte forestier.

\section{Présentation des sources}

\section{Sources écrites et iconographiques}

$5 \quad$ Parmi les sources écrites, les archives départementales du Loir-et-Cher apportent des informations précieuses sur l'administration des forêts (la série L concernant la période révolutionnaire par exemple). Elles renferment aussi des données plus précises comme les archives de la capitainerie des chasses de Chambord (série B). Aux archives nationales, la série $\mathrm{P}$ contient les registres de la Chambre des comptes de Blois, et les séries $\mathrm{K}$ et KK des actes de vente de parcelles ou de bois coupé, des ordonnances, ou des actes de dons des comtes à leurs vassaux concernant les forêts de Blois, Russy et 
Boulogne (KK, 269 à 306: comté de Blois; 299: forêts du comté). La série Q/1 concernant les forêts de Blois aux $\mathrm{XV}^{\mathrm{e}}$ et $\mathrm{XVI}^{\mathrm{e}}$ siècles renferme des procès-verbaux de ventes, une visite de forêt et le journal des eaux et forêt du comté de Blois (1562-1563). La série $\mathrm{G}^{3}$ conserve les archives de l'administration des eaux et forêts.

L'ouvrage intitulé Le Livre du roi Modus et de la reine Ratio, paru pour la première fois en 1486, est un manuel traitant des pratiques de la vénerie et de la fauconnerie qui avaient cours au $\mathrm{xv}^{\mathrm{e}}$ siècle. Le Théâtre d'agriculture et Mesnage des champs d'Olivier de Serres (1539-1619), agronome, apporte la première synthèse scientifique des techniques agricoles et des bonnes pratiques qu'un fermier gentilhomme doit mettre en place pour subvenir aux besoins de sa famille. L'ouvrage comprend également des informations sur la manière d'aménager les lieux de conservation du gibier.

7 Les travaux de Jean Martin-Demézil (thèse : « Les forêts du comté de Blois jusqu’à la fin du Xve siècle ", soutenue en 1941) (Martin-Demézil, 1963 et 1964) sont indispensables pour rassembler les informations existantes et établir un premier inventaire des sources écrites utiles. Le vicomte Joseph de Croy (1744-1839), historien chartiste, a réalisé une thèse consacrée à la Chambre des comptes de Blois. Son ouvrage Nouveaux documents pour l'histoire de la création des résidences royales en bords de Loire, paru en 1894, est encore une référence pour l'histoire de ce territoire. Il y aborde notamment la construction des châteaux de Blois et de Chambord à travers des archives de la Chambre des comptes.

Figure 2. Carte postale d'une habitation de famille dans les coupes de taillis, Loir-et-Cher

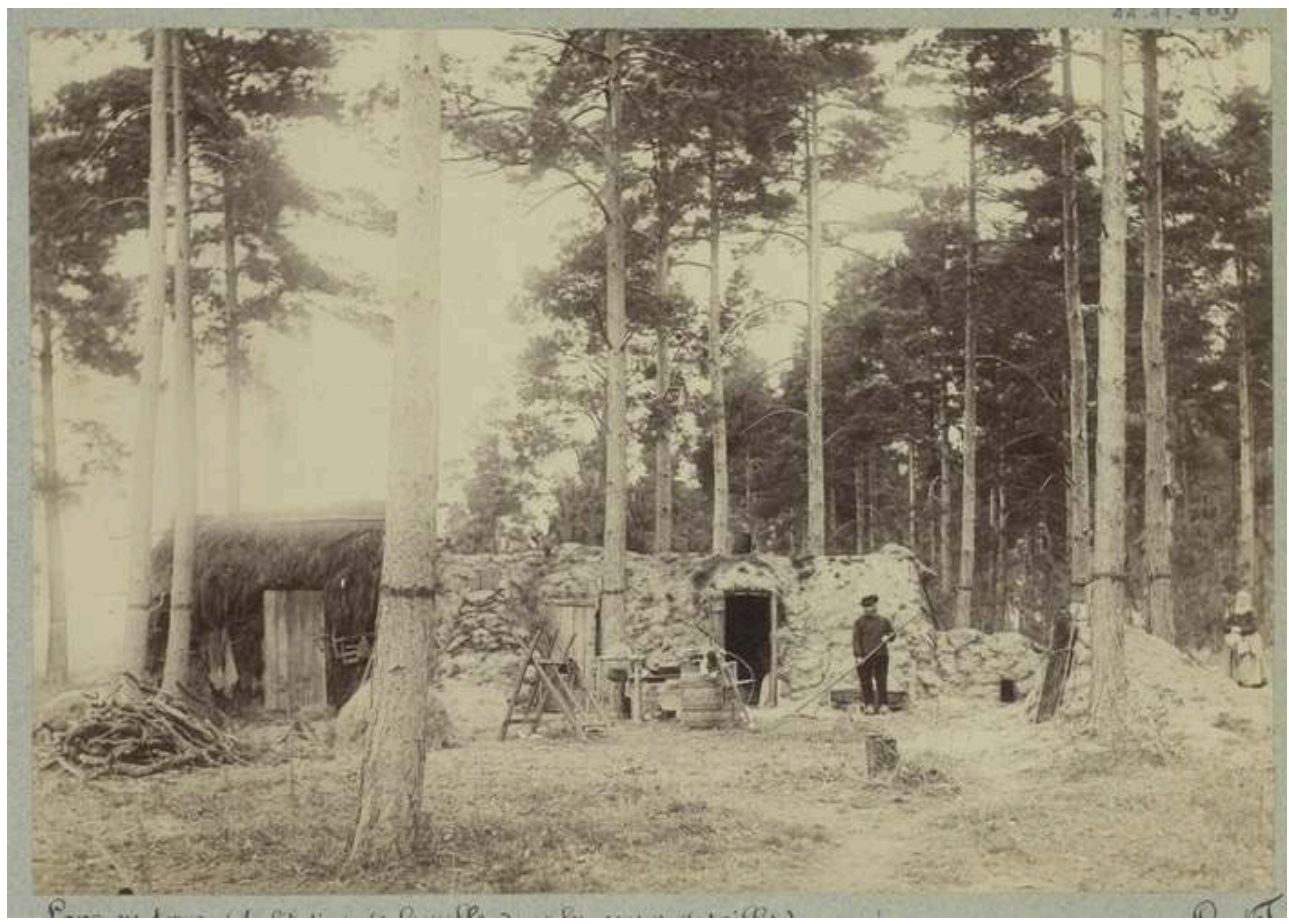

Source : Ph.1944.41.409, MuCEM.

Les sources iconographiques intègrent des vues de paysage, des scènes de la vie quotidienne (tableaux, gravures, enluminures) pouvant apporter des informations sur l'aspect du paysage ancien, les pratiques courantes. Les anciennes cartes postales du début du xxe siècle (figure 2) constituent une source ethnographique sur la vie en forêt. 
Enfin, les cartes et plans anciens informent sur l'occupation du sol et le découpage du territoire (carte de Cassini, plan terrier, cadastre...). Nous gardons à l'esprit le caractère souvent subjectif de ces plans en les contextualisant et en les croisant avec d'autres sources d'information. Ils apportent néanmoins la vision des contemporains sur leur environnement.

\section{Archives du sol}

L'archéologie a recours à des sources matérielles obtenues sur le terrain qui concernent l'environnement dans son ensemble. Il peut s'agir de sources matérielles transmises par des institutions sous forme de système d'information géographique: l'office national des forêts (ONF) procure des informations sur la gestion forestière (stations forestières, chemins d'exploitation, types et âges des essences, états des parcelles) et l'Institut géographique national (IGN) les données topographiques et administratives. Il s'agit également de données obtenues par la prospection de surface: matériel archéologique (tessons de céramique, objets en métal, matériaux de construction) pouvant éventuellement servir à dater et/ou à caractériser un site identifié.

Dans le cadre du programme SOLiDAR, des données géomorphologiques ont été collectées mais ne seront pas présentées ici. Des données pédologiques existent mais ne sont pas homogènes sur l'ensemble de la zone d'étude. Elles sont pourtant essentielles pour notre compréhension de la conservation des vestiges, elles sont donc intégrées à notre étude chaque fois que cela est possible.

\section{La technique de télédétection LiDAR}

Figure 3. Schéma d'acquisition des données LiDAR

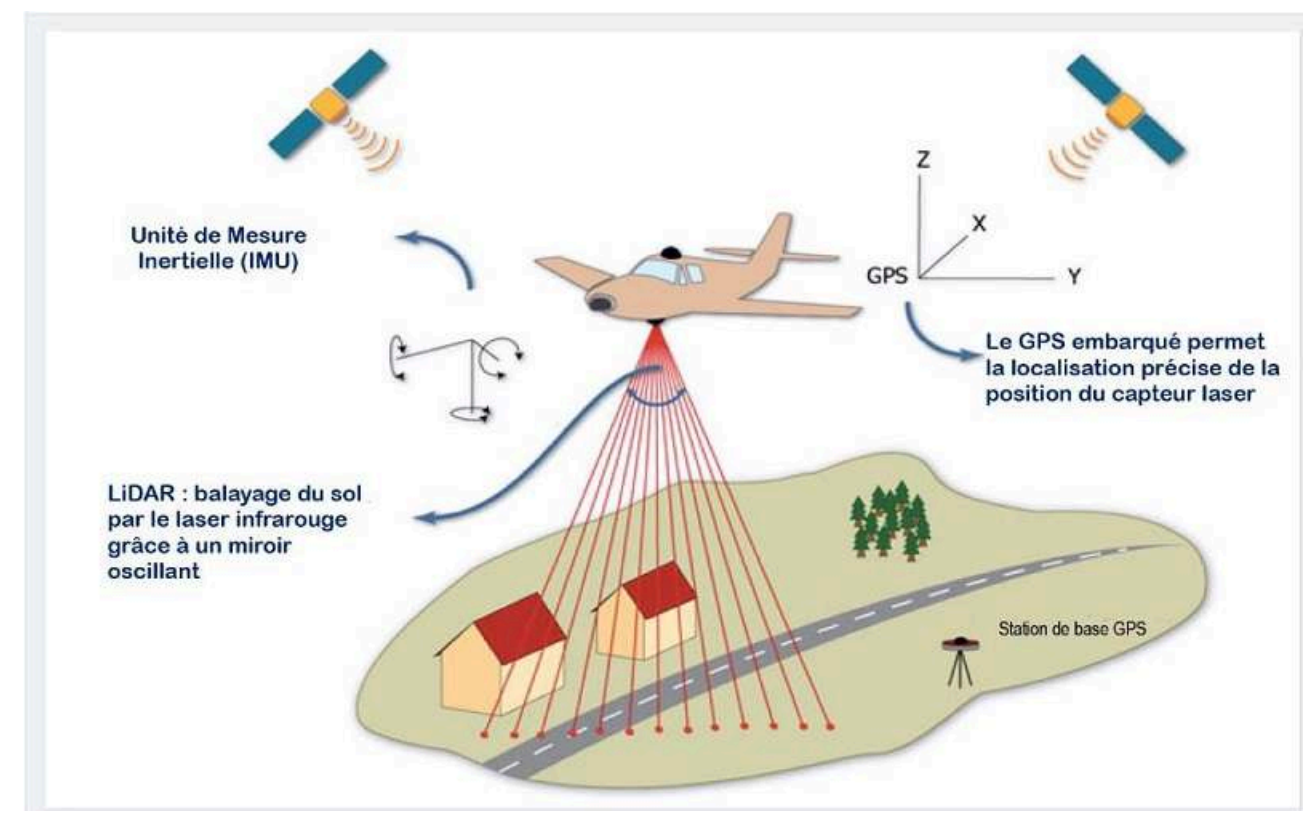

Source : Aude Crozet d'après ModeltTER/ZRC SAZU, Z. Kokalj, K. Ostir.

Le LiDAR est un dispositif de relevé microtopographique fondé sur une mesure de distance (figure 3). L'appareil est composé d'un émetteur laser, d'un miroir oscillant, 
d'un GPS, d'une unité de mesure inertielle (IMU, Inertial Measurement Unit) et est, dans ce cas, embarqué dans un avion. Le faisceau lumineux est envoyé au sol à très grande vitesse de manière itérative grâce au miroir oscillant. L'appareil détermine la localisation de tous les obstacles rencontrés par le laser à partir du temps aller-retour $\mathrm{du}$ faisceau - la vitesse de la lumière étant connue - entre le point d'impact (point réflecteur) et l'émetteur. L'IMU permet de rectifier la position en fonction du tangage de l'appareil. On obtient ainsi un nuage où chaque point possède des coordonnées (latitude, longitude, altitude). Ce nuage de points est filtré, c'est-à-dire débarrassé virtuellement $d u$ couvert forestier, pour ne garder que les points du sol. Après traitement, un modèle numérique de terrain (MNT) de haute précision est obtenu. À partir de ce MNT, plusieurs visualisations, les données dérivées (figure 4), sont produites en fonction de différents azimuts ou des critères comme la pente ou l'altitude des points, afin de mieux détecter les microvariations du relief. Celles-ci correspondent soit à des anomalies naturelles, soit à des vestiges anthropiques (parcellaires, carrières, charbonnières, habitat...).

Figure 4. Forêt de Russy
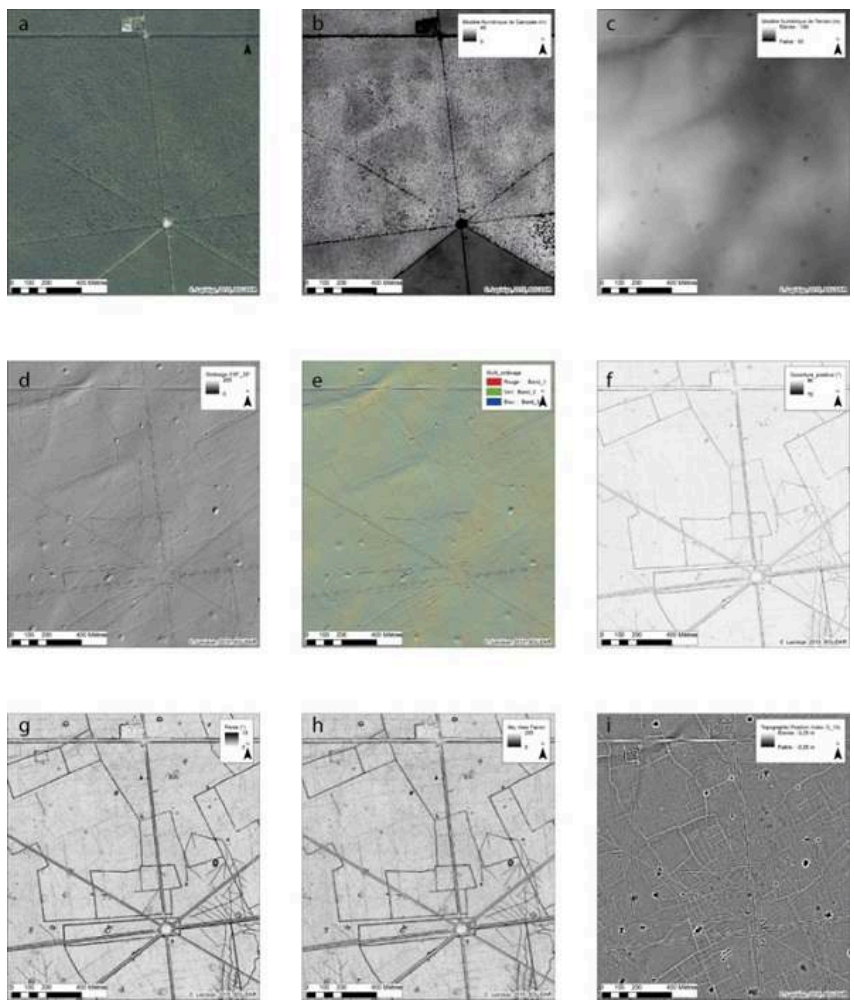

a. Orthophotographie ; b. MNC ; C. MNT ; d. Ombrage simple 315_35; e. Multi-ombrage ; f. Ouverture positive ; g. Pente; h). SVF ; i). TPI.

Sources : Clément Laplaige, 2015, IGN (a) et SOLiDAR.

12 Le principal avantage du laser aéroporté est sa capacité à pénétrer la couverture végétale pour atteindre le sol. Cette technique permet donc de faire de la détection sous couvert forestier, environnement pas ou peu propice à la prospection à vue, systématique et extensive. Utilisée depuis une quinzaine d'années en archéologie, la télédétection LiDAR a permis de renouveler la connaissance archéologique des forêts (Opitz et Cowley, 2013). 
Dans la majorité des forêts sur lesquelles des acquisitions LiDAR ont été menées, le nombre de vestiges connus a été largement augmenté (Chase et al., 2011 ; Kooistra et Maas, 2008; Nuninger et al., 2011; Risbøl et al., 2013; Sittler et Hauger, 2007). La campagne LiDAR de 2015 a permis d'obtenir des données avec une précision absolue de l'ordre du décimètre en altitude et en plan, et d'une densité moyenne de 10 points au sol par $\mathrm{m}^{2}$ (figure 5). Les résultats seront présentés en troisième partie.

Figure 5. Tableau des données LiDAR en chiffres

\begin{tabular}{|l|l|}
\hline \multicolumn{1}{|c|}{ Paramètre } & Valeur nominale \\
\hline Hauteur de vol & $600 \mathrm{~m}$ \\
\hline Vitesse de vol & 100 Nœuds \\
\hline Angle de scan & $60^{\circ}$ \\
\hline Fréquence de scan & $400 \mathrm{kHz}$ \\
\hline Précision altimétrique $(\sigma)$ & $10 \mathrm{~cm}$ \\
\hline Précision planimétrique $(\sigma)$ & $10 \mathrm{~cm}$ \\
\hline Densité moyenne de points & $40 \mathrm{pts} / \mathrm{m}^{2}$ \\
\hline Densité de points sol après classification & $11 \mathrm{pts} / \mathrm{m}^{2}$ \\
\hline Nombre de recouvrements & 3 à 4 \\
\hline
\end{tabular}

\section{Histoire de l'exploitation ancienne de la forêt à travers les sources écrites}

\section{Les premières mentions d'un agrosystème dans le comté de Blois}

L'agrosystème (Duby, 1975, p. 92) pastoral repose sur l'interaction de trois milieux (l' ager, le saltus, la silva) exploités par l'homme pour différentes utilisations. Le village, au centre de cette organisation, est entouré par «l'espace cultivé » (ager) et «l'espace pastoral ». De ce dernier, dit aussi saltus, proviennent les engrais nécessaires pour la culture. La silva - «l'espace forestier » - procure les bois de feu et de construction, le fourrage pour les bêtes (fougères) et des aliments complémentaires comme les champignons et les baies. Ponctuellement, la forêt est un vivier d'approvisionnement en viande pour la population. Elle est aussi un lieu de pacage possible du bétail. Par ses actions, l'homme entretient la forêt, et la fait perdurer. L'interaction entre ces trois environnements crée un équilibre pour la subsistance de la communauté villageoise et façonne un paysage. Les sources écrites et iconographiques attestent d'un agrosystème autour des forêts de Blois, Russy, Boulogne vraisemblablement dès le $\mathrm{xl}^{\mathrm{e}}$ siècle (MartinDemézil, 1963, p. 159-160). L'une des premières mentions rapporte que le comte de Blois Études II accorda par une charte, entre 1020 et 1037, des terres et le droit de paisson à l'abbaye de Marmoutier dans sa forêt « entre deux eaux » désignant par là les massifs de Russy et de Boulogne. 


\section{La fabrique du paysage forestier dans le comté puis dans le domaine royal : auto-organisation et planification}

15 Dès le Haut Moyen Âge, si les terres appartiennent en général au seigneur, une distinction d'usage est faite entre la foresta et la silva communis. Le terme foresta apparaît $\mathrm{au} \mathrm{VII} \mathrm{e}^{\mathrm{e}}$ siècle et est institutionnalisé à l'époque carolingienne (752-987). Il s'agit d'une vaste réserve de chasse, une mise en défens, qui relève du droit régalien et par extension du droit féodal. Au $\mathrm{x}^{\mathrm{e}}$ siècle, le terme juridique de forêt devient la simple désignation d'un espace boisé, tandis que le terme de garenne désigne un "vaste territoire de chasse clos » (Zadora-Rio, 1986, p. 133). Dans tous les cas, il s'agit d'une zone exclusivement réservée à la chasse seigneuriale, et interdite aux paysans. Plus tard, le terme de garenne est employé pour désigner des enclos aménagés pour l'élevage de petit gibier (lapins, oiseaux...) (ibid.). La silva communis est une partie du bois que le seigneur ouvre aux paysans pour les usages communs. Le paysage forestier est façonné de manière empirique en fonction des usages qu'en font les ayants droit (dans une forme d'auto-organisation), ou par une intervention de planification à plus ou moins grande échelle.

\section{Auto-organisation}

Les droits d'usages (Devèze, 1961) consentis par le seigneur sont de plusieurs ordres :

- Ramassage de matière première : l'affouage (ramassage du bois de feu), la ramée (coupe de branches d'ormes, frênes et peupliers), le marronnage (coupe des perches pour la fabrication des piquets et des outils), l'émondage (coupe de branches de bois vert pour construire des palissades), bois « à faire cendre ou charbon »... ;

- pratiques agricoles : la glandée (ou encore pacage ou panage) permettant d'amener le bétail - souvent des porcs - en bordure de forêt, l'essartage (ou culture sur brûlis) permettant de gagner du terrain cultivable sur la forêt, la feuillée (récolte de feuilles, de fougères, de genêts et d'ajoncs pour amender les sols de culture - fumure - ou servir de litière), culture de châtaigniers pour les échalas pour la vigne ;

- nourriture : ramassage de baies, de glands, de champignons, de petit gibier.

Néanmoins, ceux-ci ne sont pas tous représentés dans les sources écrites pour notre zone d'étude. En 1218 (et tout au long du XIII ${ }^{\mathrm{e}}$ siècle), un acte du comte de Blois autorise le ramassage des branches (vertes) et du bois mort hors des défens en forêt de Boulogne (Martin-Demézil 1963 : 162). Toujours dans cette forêt, dans le bois de Saint-Solenne, les moines de l'abbaye de Bourgmoyen obtiennent en 1235 droit d'essartage, de vente, de chasse de petits animaux alors que le comte se réserve toujours la chasse des grands animaux (original : AN, K 1207, $\mathrm{n}^{\circ} 10$; copie du XVI $\mathrm{e}^{\mathrm{e}}$ siècle aux AD 41, 3 h 61, $\mathrm{n}^{\circ} 1$ ).

Par ailleurs certaines pratiques ont laissé des traces dans le paysage que nous étudions, sans pour autant être datées par les textes:

- La fabrication de charbon. Une meule en forme de dôme est construite en empilant des morceaux de bois puis recouverte d'une couche de terre et de mousse afin d'éviter le passage de l'air. Une cheminée ménagée au centre permet l'allumage de la meule. La combustion se fait sous atmosphère réductrice, c'est-à-dire sans oxygène, pour ne garder que le carbone du bois : le charbon; elle dure plusieurs jours, voire plusieurs semaines, en fonction de la taille de la meule. Pour ramasser le charbon, la meule est ratissée du centre vers l'extérieur. Une trace au sol, la plupart du temps ronde, est repérable par la présence de résidus de charbon. 
La très grande quantité de ces "plateformes » de charbonniers dans les forêts considérées atteste bien de cette pratique mais la datation, qui serait longue et périlleuse, n'est pas envisagée pour le moment. Durant tout le processus, la vie domestique du charbonnier, et souvent de sa famille, s'organise autour de la plateforme de charbonnage, impliquant des pratiques vivrières en forêt.

- L'extraction de minerai de fer. En forêt de Boulogne, où le minerai est parfois présent à faible profondeur, des ferriers (des amas de scories de minerai de fer, issues de la réduction du minerai) ont été repérés en prospection pédestre. La plupart sont situés à proximité de trous, qui pourraient être des fosses d'extraction du minerai (Magiorani, 1999). Les ferriers accompagnent parfois des fours temporaires, en argile, qui sont ensuite détruits. En effet, l'exploitation d'une zone pour la production de charbon, de fer ou de verre est accordée pour un temps limité.

\section{La planification}

19 La foresta est le lieu de chasse privilégié du seigneur ou du roi. Il s'agit avant tout de posséder un espace boisé conséquent, mais l'aménagement de cet espace se complexifie avec le temps.

Dès le $\mathrm{XI}^{\mathrm{e}}$ siècle, les forêts du comté de Blois étaient morcelées et grevées de très nombreux droits d'usage, parfois accordés à outrance par les comtes (Martin-Demézil, 1964). À partir du XIII ${ }^{\mathrm{e}}$ siècle, les comtes de Blois ont cherché à reconstituer un domaine forestier comtal vaste et cohérent dans les forêts de Boulogne et Russy. Comme le rappelle Jérôme Buridant « la vénerie est consommatrice d'espace [...] » (2005, p. 18-19). Rappelons que la poursuite à cheval devait revêtir le caractère de loisir et d'aventure recherché par les nobles, et faire écho au courage et à la vaillance du guerrier.

21 La forêt de Boulogne, plus éloignée de la ville de Blois, était particulièrement giboyeuse (Martin-Demézil, 1963, p. 158) et abritait les manoirs fortifiés de Montfraut et de Chambord, avant tout utilisés comme relais de chasse par les comtes de Blois (Croy, 1894). De plus, la forêt de Boulogne constituait, jusqu'à la construction du mur d'enceinte du parc de Chambord, le plus vaste espace boisé à proximité de Blois, idéal pour la chasse à courre. En ce qui concerne l'ordre de grandeur, Jean Martin-Demézil estime la superficie de Boulogne de 12500 à 13000 arpents au XVI siècle, contre 6450 à Russy et 5400 à Blois (Martin-Demézil, 1963, p. 143). Certaines transactions réalisées par les comtes de Blois nous sont parvenues, en dépit de sources assez lacunaires :

- En mai 1234, Pierre Assali reçoit une pièce de bois contre ses droits en forêt de Boulogne. Les clauses stipulent que le comte a droit de haute justice et de chasse exclusif (BNF, lat 10108 , $\left.\mathrm{n}^{\circ} 55\right)$;

- En 1255, Jean de Vendôme et sa femme Adèle vendent à Jean de Châtillon, comte de Blois, " tot lor bois qu'il avoient en Boloigne ", c'est-à-dire 140 arpents. Le comte achète tous les herbages, toute la chasse, les bêtes mortes, toute la seigneurie et la justice pour une somme de 300 livres tournois, une rente annuelle de 10 livres tournois assignée sur le four de Vienne (BNF, lat. 10108, $\mathrm{n}^{\circ}$ 75, 1255, décembre).

$22 \mathrm{Au} \mathrm{XVII}{ }^{\mathrm{e}}$ siècle, les forêts royales sont quadrillées d'allées rectilignes destinées à la chasse à courre. Ces grandes transversales se croisent à des carrefours, ou étoiles, qui permettent le rassemblement des équipages. C'est à partir de ces étoiles que les invités du seigneur ou du roi assistaient au spectacle et suivaient le parcours du gibier. Dans le parc de Chambord, les grandes allées ont été pour la plupart mises en place par le maréchal de Saxe à partir de 1745 (Chatenet, 2001). Ces grandes perspectives qui 
partent du château, et la forêt autour, sont inscrites au patrimoine de l'Unesco depuis 1981, classées monument historique depuis 1997 et font partie intégrante de la mise en valeur paysagère, particulièrement sensible à Chambord (Aménagement forestier de Chambord, 2015-2034).

Outre la chasse à courre, réservée à une élite, les hommes s'approvisionnent en viande grâce à des dispositifs spécifiques :

- Les parcs à gibier. Aux XI et XII ${ }^{e}$ siècles, il s'agit d'une zone, entourée d'une ou deux lignes de terrassement et d'une palissade. Ces parcs sont souvent boisés, et contiennent aussi des étangs ou des viviers. Le bois prend plutôt la forme d'une futaie, car les nombreux animaux enfermés consomment les strates arbustive et herbacée. Les parcs pouvaient être construits ex nihilo, ou bien un bois préexistant était entouré d'une clôture, voire d'un mur d'enceinte, quand on avait les moyens. Le premier parc à gibier mentionné est celui de Philippe Auguste, à Vincennes, construit en 1183 (Zadora-Rio, 1986). Dans le domaine de Chambord, deux parcs à gibier sont attestés sur le plan dit plan Polignac, datant du XVIII e siècle (AN NII Loir-et-Cher 2). Cinq autres enclos du même type ont également été repérés grâce aux données LiDAR. Il s'agit dans ce cas de quadrilatères, délimités par un talus intérieur et un fossé extérieur afin d'éviter la fuite du gibier.

- Les mottes à conils. Le Théâtre d'agriculture et mesnage des champs d'Olivier de Serres constitue la première description, très précise, d'une motte à conils idéale. Elles ont également été bien étudiées par Élisabeth Zadora-Rio (1986) : une motte à conils, ou terrier, ou murger à conils, doit être suffisamment aérée et isolée de l'humidité du sol. Elle doit être entourée d'un fossé intérieur en eau, où l'on pourra mettre des poissons, et d'un talus extérieur, afin d'éviter la fuite des animaux. Enfin, elle doit être plantée d'arbres et arbustes, pour l'alimentation autonome et la reproduction des lapins. Ceux-ci ne furent pas domestiqués avant le XIX ${ }^{e}$ siècle, mais bien élevés à l'état sauvage dans ces garennes dès le XIII ${ }^{e}$ siècle. De manière générale, ces mottes sont des tertres de forme elliptique et d'une taille importante, de telle sorte qu'elles ont pu être confondues avec des tumulus (Fraysse, 1961). La plus grande qui ait été répertoriée, la motte à conils de Marigné (Maine-et-Loire), est une plateforme elliptique de $30 \mathrm{~m}$ de long, sur $1 \mathrm{~m}$ d'épaisseur, et entourée d'un fossé de 1 à $2 \mathrm{~m}$ de large. De nombreux tertres de ce type ont été repérés dans le parc de Chambord, en forêt de Boulogne et en forêt de Russy.

En plus de ces constructions qui modifient le paysage forestier, l'activité cynégétique implique un aménagement spécifique de la forêt prenant en compte les besoins des animaux : nourriture et points d'eau. D'ailleurs, le terme d'aménagement, issu du vieux français «mesnaige" ou "mesnage ", est, jusqu'au xvIII ${ }^{\mathrm{e}}$ siècle, exclusivement forestier. Il s'agit des coupes, des sélections de réserves (Buridant, 2005, p. 22-23) et pour la chasse à courre, de l'entretien d'une forêt en futaie (souvent enrichie d'arbres fruitiers comme le chêne, le hêtre, le châtaignier, ou le merisier). Notons que les forêts domaniales que nous étudions ont gardé un caractère purement cynégétique (et de consommation locale du bois) tout au long de l'Ancien Régime, quand d'autres massifs forestiers du roi étaient exploités pour l'architecture ou la construction navale (en Normandie par exemple : Devèze, 1961, p. 69).

La planification de l'espace forestier concerne également les concessions accordées à des communautés religieuses ou à des vassaux laïcs à des fins d'installation et de culture. Dans ces cas, les parcelles concédées sont délimitées par des fossés, des talus, des palissades qui créent des enclos ex nihilo. En 1202, Louis $\mathrm{I}^{\text {er }}$ de Blois accorde au prieuré de Grandmont, situé dans Boulogne depuis 1163, des terres que les moines 
entourent d'un fossé. Plus tard, se trouvant à l'étroit, les moines occupèrent finalement une partie plus grande de la forêt, entourée elle aussi de fossés (Martin-Demézil, 1963, p. 162). Cette situation particulière relève autant de la planification, avec la délimitation physique d'une zone, que de l'auto-organisation, avec l'agrandissement empirique de cette zone au cours du temps. Les fossés de délimitation réalisés par les moines sont encore identifiables sur le terrain et sont représentés sur le plan datant de 1671 conservé aux Archives du Loir-et-Cher (figure 6).

Figure 6. «Plan très exact du prieuré de Boulogne » en 1671

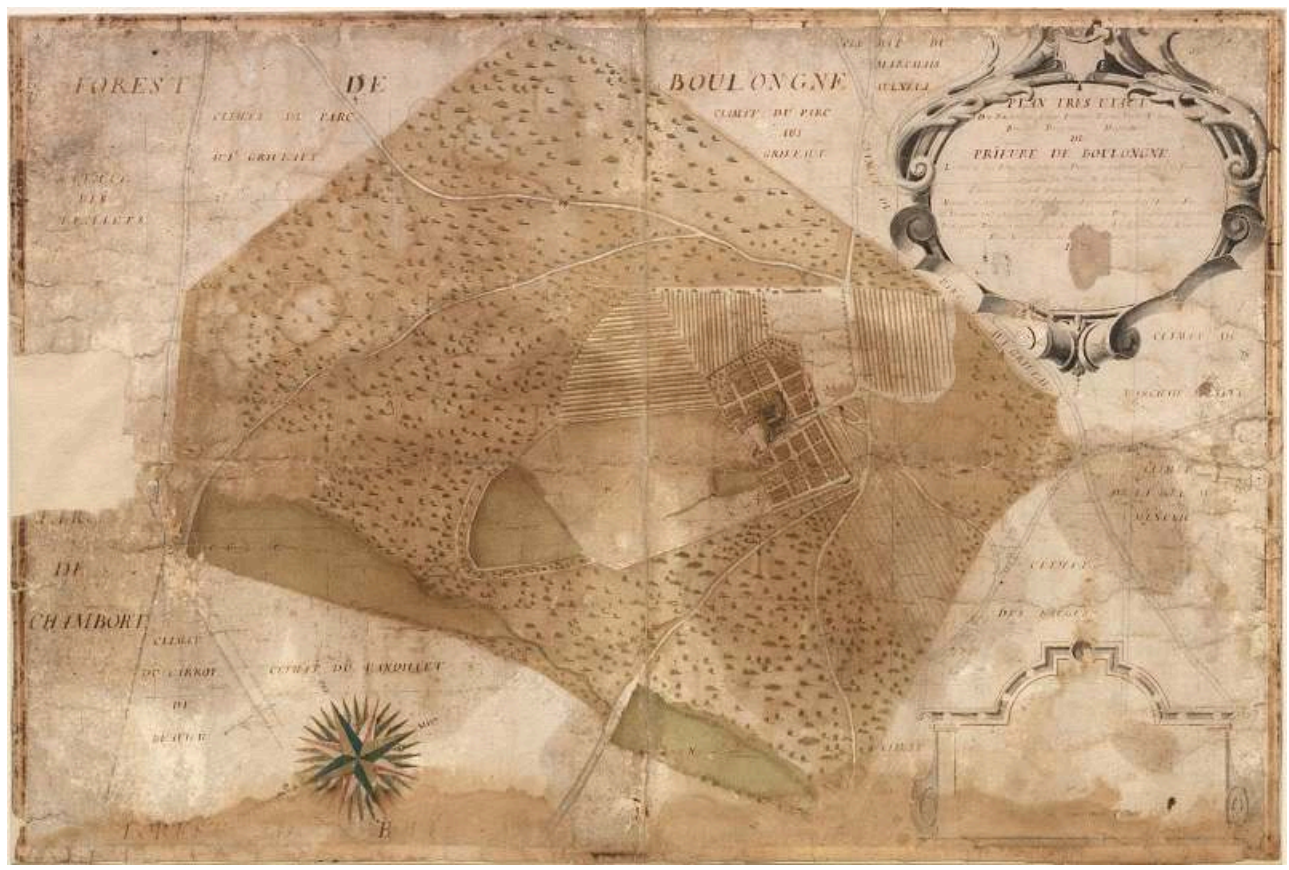

Source : Archives du Loir-et-Cher, 1Fi-1585

L'étude des sources écrites et iconographiques permet de comprendre la pression anthropique sur l'écosystème forestier. Dans la zone d'étude SOLiDAR - et notamment dans le domaine de Chambord, dont le caractère clos rend plus visible l'impact des herbivores sur la flore - certains paysages évoquent des zones de pacage médiévales : grande prairie clairsemée d'arbres majestueux (figure 7). Ces zones de forêt dite ouverte se caractérisent par l'absence quasi totale de strate arbustive, consommée par les animaux, et des arbres aux longs fûts - débarrassés des branches basses par les cervidés - et au houppier très développé. Cette identification de "paysages témoins » est intégrée à l'approche archéologique du paysage. Elle permet, d'une part, d'imaginer l'aspect de paysages passés (proches ou lointains), d'autre part, dans une perspective patrimoniale, d'observer la continuité ou la disparition de certains paysages. 
Figure 7. Paysage évoquant une zone de pacage médiévale. Prairie de la princesse Zita, domaine national de Chambord

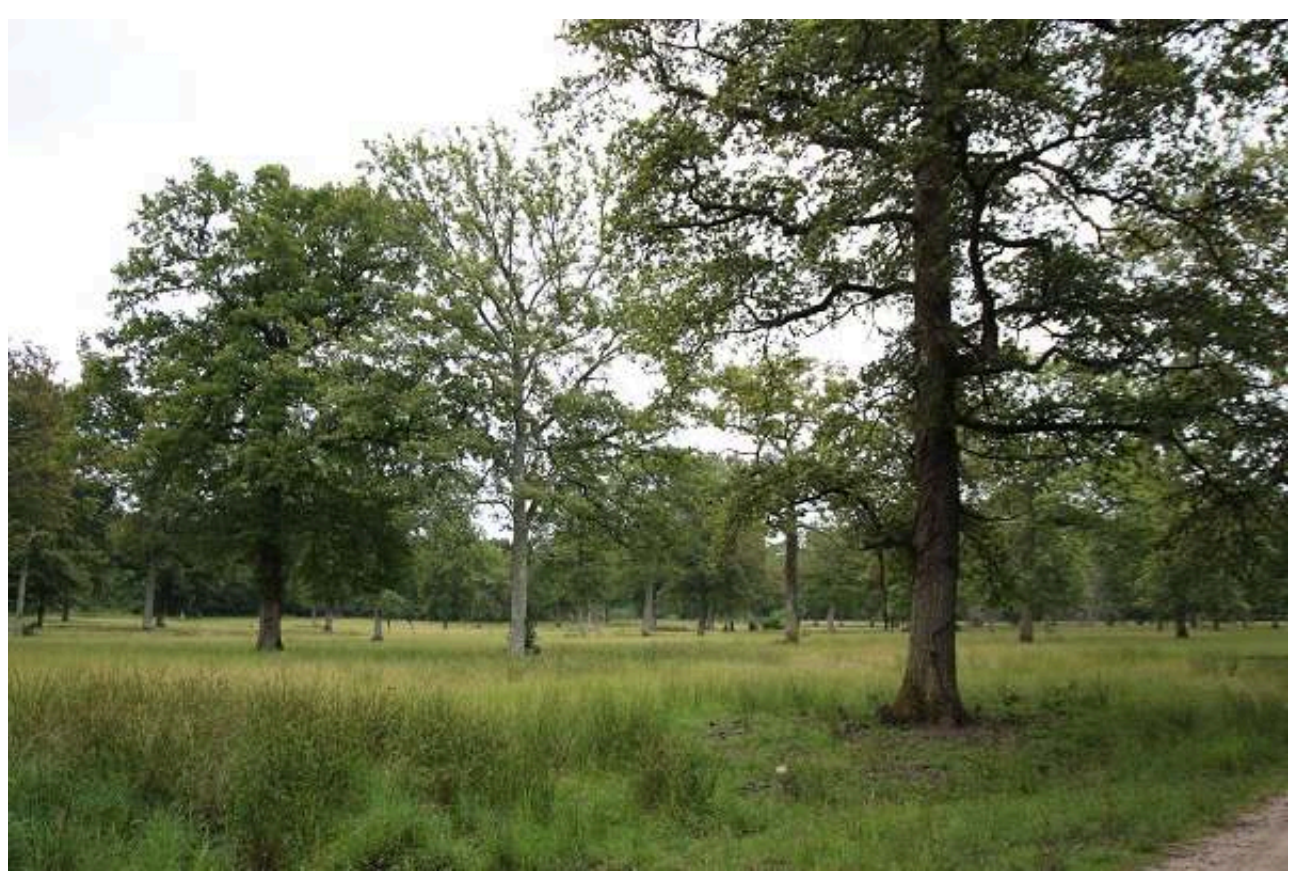

Source : Aude Crozet, 2016, SOLiDAR.

\section{Les traces d'organisation du territoire antérieur à la forêt : l'apport du LiDAR}

\section{Présentation des résultats}

Une partie des forêts de la zone d'étude, en particulier la forêt de Boulogne, le parc de Chambord et la forêt de Russy, a été sillonnée pendant 15 ans par un prospecteur amateur, Louis Magiorani. De nombreux vestiges ont ainsi été repérés et ajoutés à l'inventaire archéologique du Service régional d'archéologie : tertres, ferriers, limites parcellaires, habitats... (figure 8). L'opération LiDAR menée en 2015 a permis d'étendre la détection des vestiges archéologiques à la forêt de Blois. Cette opération a eu pour intérêt premier de couvrir une grande surface forestière très rapidement et de disposer, après traitement, manipulation et vérification des données, d'une cartographie détaillée des anomalies microtopographiques d'origine anthropique. 
Figure 8. Carte des vestiges repérés en prospection pédestre et inventoriés dans la base de données du patrimoine archéologique patriarche

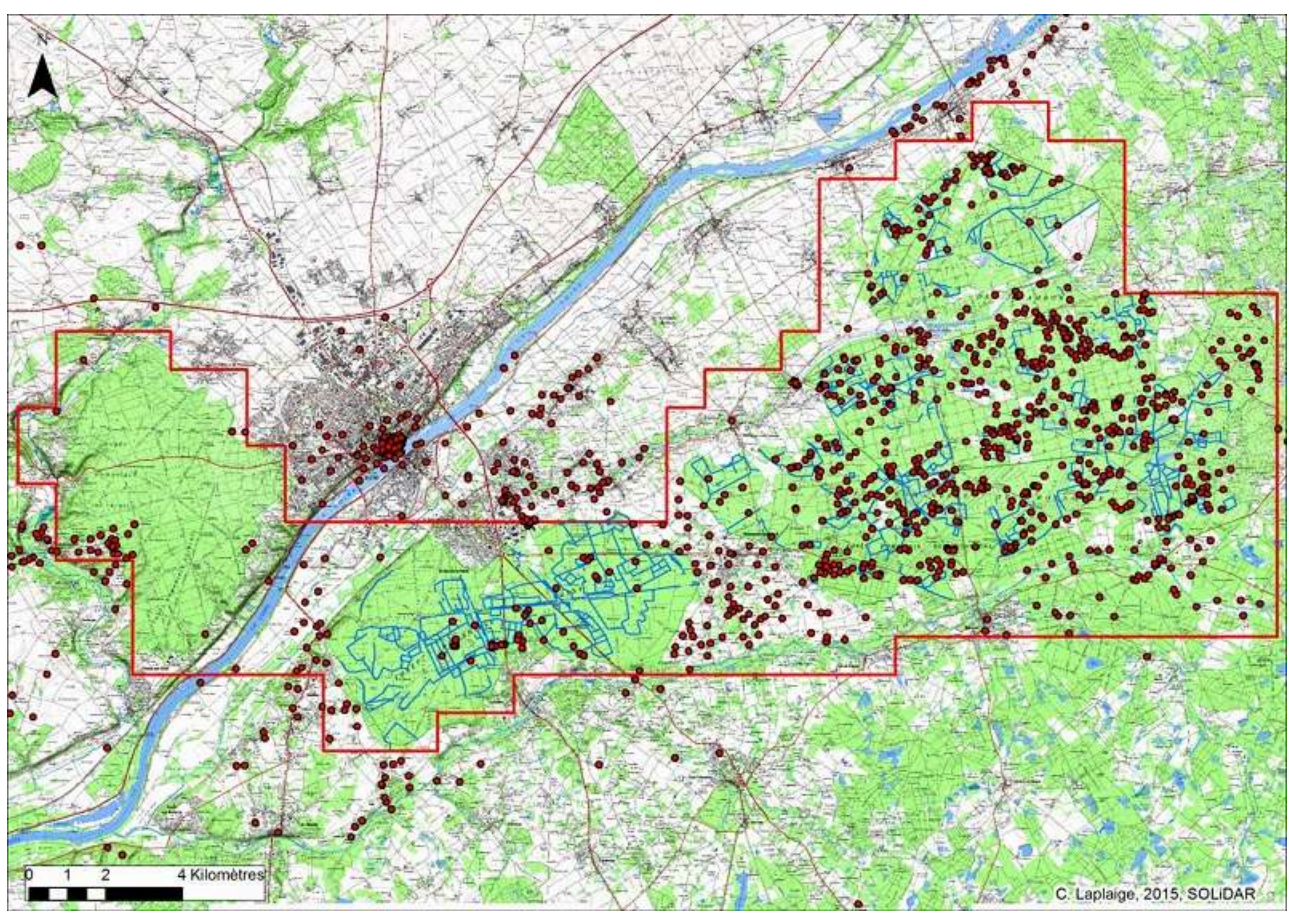

Source : Clément Laplaige, 2015, SOLiDAR

Les résultats obtenus concernent deux aspects de la dynamique de peuplement.

- Ils ont révélé l'existence d'une occupation antérieure à la forêt. Cette occupation se caractérise par la présence d'habitats, de voies, de champs bombés ${ }^{2}$ et en particulier des limites parcellaires formant des modules de petite surface (1,5 ha en moyenne), suggérant ainsi un paysage ouvert.

- Ils ont mis en exergue les traces de pratiques sylvicoles (loges de forestiers, charbonnières par exemple) qui, pour certaines, n'étaient pas visibles à l'œil nu.

\section{Les différentes trames parcellaires}

\section{Résultats}

Les premières analyses des données LiDAR ont montré qu'il existait, dans la zone d'étude, au moins trois trames parcellaires se superposant dans la quasi-totalité des massifs de Blois, Russy, Boulogne et Chambord. L'extension de ces trois trames est observable sur la figure 9. 
Figure 9. Carte des linéaments parcellaires détectés dans la zone d'étude superposés au parcellaire forestier actuel

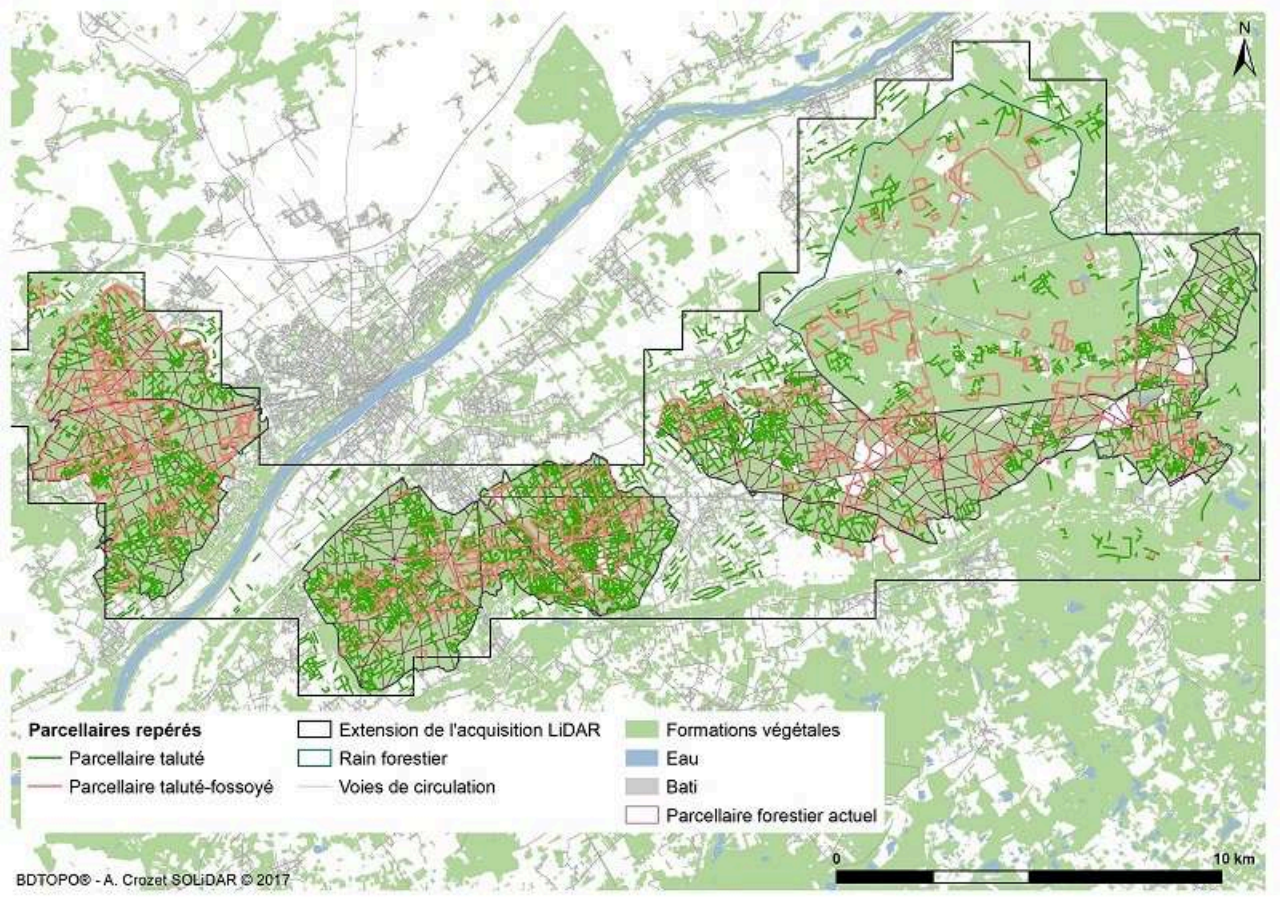

Aude Crozet, 2017, SOLiDAR.

Le modèle dérivé des données LiDAR (figure 10a) permet d'observer la superposition des 3 trames, listées ci-dessous de la trame supérieure à la trame inférieure. 
Figure 10. Zoom sur les 3 types de parcellaires en forêt de Russy, la « Croix Pineau »
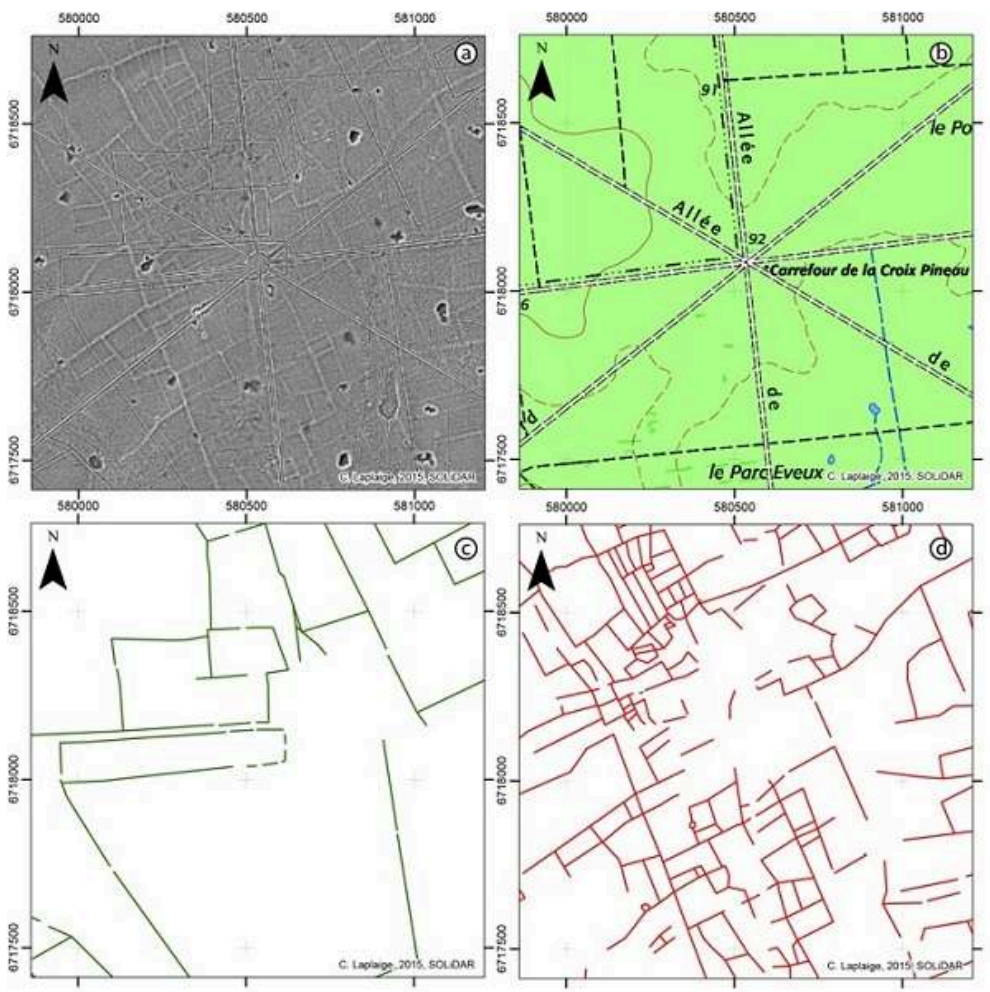

a : Scène LiDAR ; b : Scan25 ; c : Système taluté-fossoyé ; d : Système taluté Sources : Clément Laplaige, 2015, SOLiDAR (a,c et d) ; IGN (b).

- Au premier plan le système viaire de la trame parcellaire actuelle (figure 10b).

- Un système composé de talus et de fossés (figure 10c). Cette trame a été identifiée par les prospections pédestres de Louis Magiorani. Ces linéaments, qui représentent $320 \mathrm{~km} \mathrm{de}$ longueur cumulée, s'étendent de part et d'autre de la Loire et sont représentés dans tous les massifs concernés par le programme. Ils dessinent une trame constituée de modules rectangulaires, carrés, polygonaux, enchâssés ou non. En coupe, la structure talus-fossé mesure en moyenne de 6 à $8 \mathrm{~m}$ de large pour une profondeur inférieure au mètre. Cette forme de limite parcellaire est recoupée par la trame viaire contemporaine mais peut encore servir de délimitation des forêts actuelles.

- Un système composé uniquement de talus (figure 10d). Il n'avait jamais été reconnu en prospection. En effet, les anomalies du microrelief sont bien trop ténues pour être observées à l'œil nu. La figure 11 montre ces talus sur les données LiDAR, sous forme de linéaments blancs bien nets comparés à leur visibilité très relative sur le terrain. Les talus les plus marqués mesurent environ 15 centimètres de haut pour une quinzaine de mètres de large. Cette trame se développe sur les quatre massifs forestiers, soit environ $90 \mathrm{~km}^{2}$ (respectivement Blois $22 \mathrm{~km}^{2}$, Russy $33 \mathrm{~km}^{2}$, Boulogne $22 \mathrm{~km}^{2}$ et Chambord $9 \mathrm{~km}^{2}$ ). Ce système semble, à première vue, assez homogène, orienté perpendiculairement à l'axe de la Loire (qui est à peu près orienté à $45^{\circ}$, c'est-à-dire nord-est/sud-ouest) avec des parcelles ayant une surface majoritairement de l'ordre de l'hectare. Toutefois, le système n'est pas continu: il s'interrompt dans la plaine alluviale de la Loire et au niveau de Mont-prèsChambord, entre les forêts de Russy et de Boulogne. Enfin, la partie est de la zone d'étude est beaucoup moins riche de ce type de vestiges (figure 9). 
Figure 11. La trame parcellaire composée de talus, détectable (linéaments blancs) sur les données dérivées LiDAR, est plus difficile à repérer sur le terrain

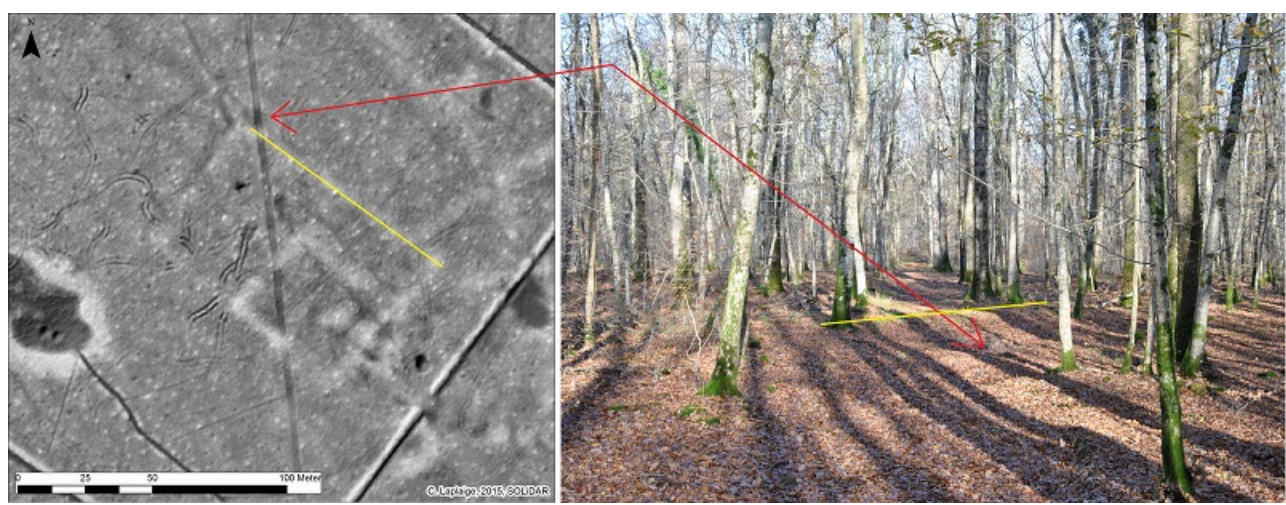

Sources : LiDAR : Clément Laplaige, photographie : Aude Crozet, 2015, SOLiDAR.

\section{Premières interprétations des trames parcellaires} peut-il également être observé en zone ouverte ? Cela correspond-il à un changement de type d'occupation? Il est couramment admis que des parcellaires anciens peuvent avoir perduré jusqu'à l'époque moderne car ils sont des éléments structurels forts du paysage (Chouquer, 2000). L'analyse particulière d'un secteur au nord de la forêt de Boulogne, sur la commune de Huisseau-sur-Cosson, apporte des informations sur la continuité de cette trame en zone ouverte. La figure 12 montre que le système de talus se retrouve aussi à l'extérieur du massif de Boulogne (figures 12a et 12c). Les vestiges 
de ces talus provoquent encore des anomalies phytographiques ${ }^{3}$. La figure $12 \mathrm{~b}$ montre ces traces linéaires plus claires dans la zone cultivée. La superposition du cadastre napoléonien (daté pour l'arrondissement de Blois de 1808-1838) à l'image LiDAR (12d) indique que ces talus correspondent à de la trame viaire et à des limites de carrés de culture. Cet exemple permet de poser l'hypothèse de la pérennité de ce système parcellaire jusqu'au XIX ${ }^{\mathrm{e}}$ siècle alors qu'il est fossilisé par la mise en place des massifs forestiers. Il reste à tenter d'en déterminer l'origine.

Figure 12

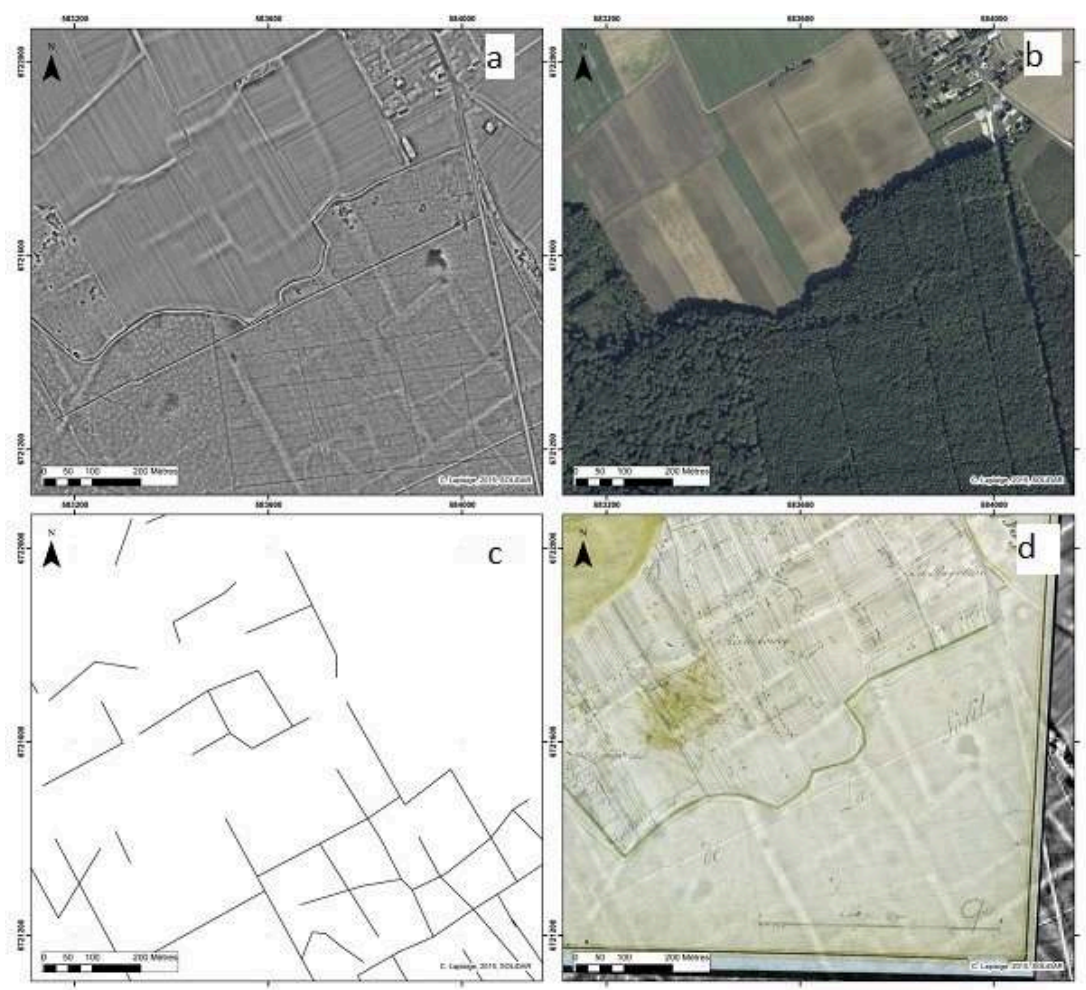

a. Scène LiDAR révélant des talus sous le couvert boisé et en zone ouverte ; b. Orthophotographie où les talus correspondent à des anomalies phytographiques ; c. Schéma d'interprétation ; d. Plan cadastral napoléonien drapé sur les données LiDAR. Huisseau-sur-Cosson (41), le « Richebourg ».

Sources : Clément Laplaige, 2015, SOLiDAR.

\section{Les limites de la datation par la prospection}

La recherche d'habitats connectés au parcellaire est la manière la plus courante de dater ce dernier (Georges-Leroy et al., 2009). La manière dont des sites ont été conservés par la forêt et leur morphologie visible sur les données LiDAR ont été largement documentées notamment lors des acquisitions LiDAR sur la forêt de Haye (54) de Besançon (25) ou du Châtillonnais (21) (Georges-Leroy et al., 2009 ; Laplaige, 2012). Nous sommes partis de ce principe pour tenter de dater le parcellaire constitué de talus.

Pour cela, nous avons sélectionné des sites d'habitats repérés par Louis Magiorani lors de ses prospections et des ensembles de talus pouvant trahir la présence d'habitats connectés au parcellaire. En somme, la recherche a porté sur de petites parcelles, inférieures à 1 ha, incluant de préférence un tertre (figure 13). Un corpus de six sites a été constitué. À la prospection, tous ont livré un peu de céramique très fragmentée 
dans les chablis ${ }^{4}$, les taupinières ou en retournant les feuilles. La majorité de la céramique d'un habitat prospecté date du Haut Empire ( $\mathrm{I}^{\mathrm{er}}$ siècle av. J.-C.- $\mathrm{III}^{\mathrm{e}}$ siècle ap. J.-C.).

Figure 13. Scène LiDAR révélant un probable habitat, pointage des découvertes de mobilier et représentation en coupe des talus et de l'élévation centrale

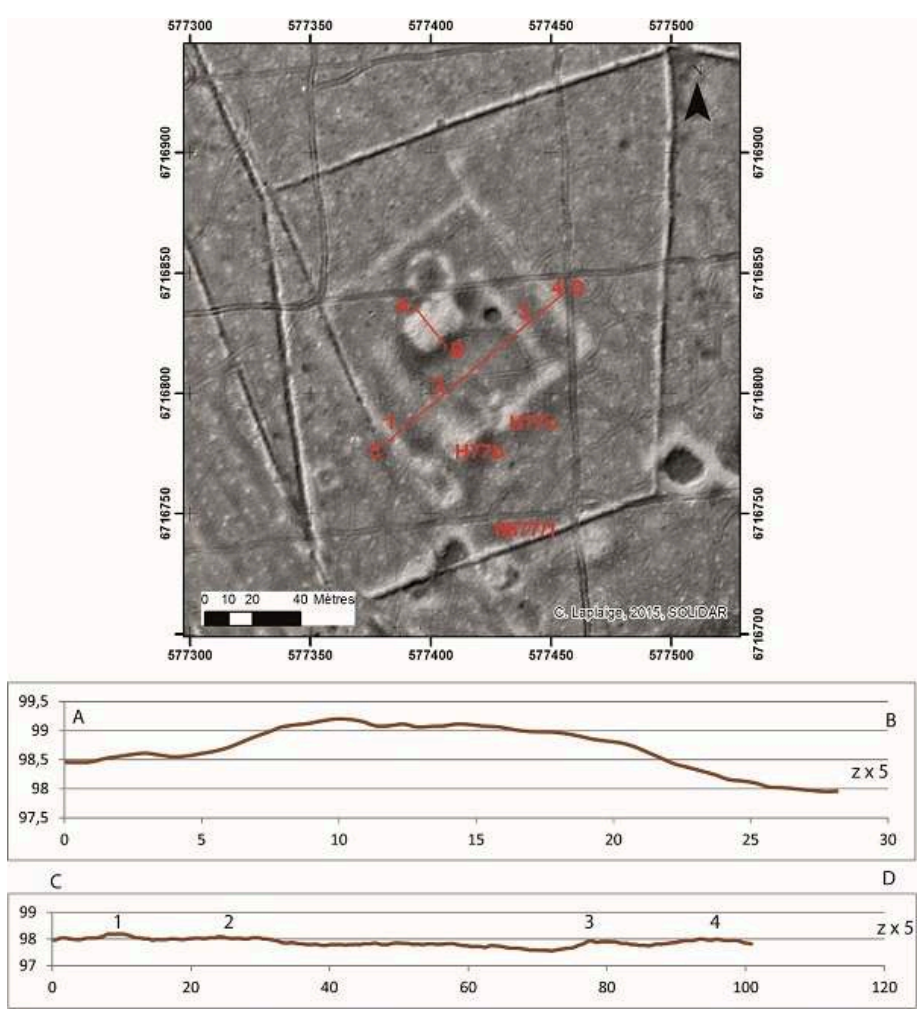

Sources : Clément Laplaige, 2015, SOLiDAR.

37 Cependant, cela ne permet pas d'aller plus loin que la simple hypothèse d'une occupation à cette période. Par ailleurs, pour parvenir à la datation d'un parcellaire, d'autres écueils sont à prendre en compte :

- la datation d'un habitat ne fournit pas les bornes chronologiques du parcellaire auquel il est relié. En effet, ce dernier a pu être en place avant l'habitat et perdurer après son abandon ;

- la forte fragmentation et la faible abondance du mobilier, en particulier de la céramique, issu des prospections, limitent les interprétations chronologiques et fonctionnelles ;

- le parcellaire s'étendant sur $100 \mathrm{~km}^{2}$, il faut se garder de généraliser les observations à partir de quelques analyses ponctuelles.

\section{Conclusion}

Les premiers résultats du programme présentés ici valident l'intérêt d'une démarche multidisciplinaire pour la compréhension de l'histoire du paysage. L'étude des sources écrites et iconographiques permet de mettre en évidence des pratiques qui ont façonné le paysage. Le croisement des disciplines permet de répondre aux questions de fonctionnalité des vestiges. Les différentes fonctionnalités sont traduites par les diverses morphologiques des parcellaires observés. La lecture du paysage est ainsi 
abordée par l'étude des pratiques anciennes (auto-organisées ou planifiées) ellesmêmes objets d'étude de l'archéologue.

L'absence d'archives écrites pour les périodes les plus anciennes laisse une carence d'informations sur l'occupation du sol, que vient combler le LiDAR par la découverte d'un parcellaire attestant d'un paysage ouvert et probablement agricole précédant la forêt. De nouvelles questions sont alors soulevées: de quand datent ces limites parcellaires inédites? Quels sont les phénomènes à l'œuvre dans leur élaboration ? À partir de quand voit-on apparaître la forêt dans le comté de Blois? Et de nouvelles perspectives envisagées : cette zone géographique aux confins de la Sologne renferme des éléments du paysage que nous n'avons pas abordés ici : les mares, l'hydrologie en général, les défrichements, les voies de communication...

Les recherches archivistiques, la prospection et la télédétection LiDAR pourront être enrichies d'approches supplémentaires. La datation par thermoluminescence pourrait répondre aux questions de datation des talus observés ou détectés. L'ethnographie apporterait des connaissances sur les pratiques sociales anciennes héritées. L'écologie, l'archéobotanique compléteraient la compréhension que nous avons de la conservation des vestiges en milieu forestier dans une optique de conservation du patrimoine tant naturel que culturel.

\section{BIBLIOGRAPHIE}

Aménagement forestier, Domaine national de Chambord, ONF, Direction territoriale CentreOuest - Auvergne Limousin, Boigny-sur-Bionne, 2015-2034, URL : http://www.loir-etcher.gouv.fr/Actualites/Document-d-amenagement-forestier-2015-2034-du-domaine-nationalde-Chambord.

Buridant, J. « La gestion des forêts de vénerie au XVII ${ }^{\mathrm{e}}$ siècle ", Dix-Septième Siècle, $\mathrm{n}^{\circ}$ 226, Paris, PUF, 2005/1, p. 17-27.

Carpentier, V. et Marcigny, C. (dir.), Des hommes aux champs. Pour une archéologie des espaces ruraux du Néolithique au Moyen Âge, Rennes, Presses universitaires de Rennes, 2012.

Chalvet, M., Une histoire de la forêt, Paris, Éditions du Seuil, 2011.

Chase, A., Chase, D., Weishampel, J., Drake, J., Shrestha, R., Clint Slatton, K., Awe, J., Carter, W., " Airborne LiDAR, Archaeology, and the Ancient Maya Landscape at Caracol, Belize ", Journal of Archaeological Science, 38 (2), 2011, p. 387-98, URL : http://www.caracol.org/wp-content/uploads/ 2016/05/ChaseEtAl2011.pdf et doi :10.1016/j.jas.2010.09.018.

Chatenet, M., Chambord, Paris, Éditions du Patrimoine, 2001.

Chouquer, G., Favory, F. « Aux origines antiques et médiévales des parcellaires », Histoire et Sociétés rurales, $\mathrm{n}^{\circ} 4,1995$, p. 11-46.

Chouquer, G., L'Étude des paysages. Essai sur leurs formes et leur histoire, Paris, Errance, 2000. 
Croy, J. de, « Nouveaux Documents pour l'histoire de la création des résidences royales en bords de Loire »., thèse de doctorat, Paris, Picard et Fils, 1894.

Dambrine, E., Dupouey, J.-L, Laüt, L., Humbert, L., Thinon, M., Beaufils, T., Richard, H., « Present Forest Biodiversity Patterns in France Related to Former Roman Agriculture », Ecology, 88 (6), 2007, p. 1430-1439, doi :10.1890/05-1314.

Deforce, K., Boeren, I., Adriaenssens, S., Bastiaens, J., Keersmaeker, L. de, Haneca, K., Tys, D., Vandekerkhove, K., « Selective Woodland Exploitation for Charcoal Production. A Detailed Analysis of Charcoal Kiln Remains (Ca. 1300-1900 AD) from Zoersel (Northern Belgium) », Journal of Archaeological Science, 40 (1), p. 681-689, doi :10.1016/j.jas.2012.07.009

Derex, J.-M., La Mémoire des forêts, à la découverte des traces de l'activité humaine en forêt à travers les siècles, Paris, Ulmer, 2013.

Devèze, M., « La Vie de la forêt française au XVI ${ }^{\mathrm{e}}$ siècle ", thèse de doctorat, 1961, École pratiques des hautes études, Paris, SEVPEN.

Doyen, B., Decocq, G., Thuillier, P., « Archéologie des milieux boisés en Picardie », Revue archéologique de Picardie, $\mathrm{n}^{\circ}$ 1, 2004, p. 149-164, URL : http://www.persee.fr/doc/ pica_0752-5656_2004_num_1_1_2391 et doi : 10.3 406/pica.2004.2391.

Duby, G. (dir.) et Wallon, A. (dir.), Histoire de la France rurale, Paris, Éditions du Seuil, coll. « Histoire », 1975, vol. 1 et 2.

Dupouey, J.-L., Sciama, D., Koerner, W., Dambrine, E., Rameau, J.-C., « La végétation des forêts anciennes ", Revue Forestière Française (Rev. For. Fr.), nº 54, 2002, p. 521-32.

Dupouey, J.-L., Dambrine, É., Dardignac, C., Georges-Leroy, M., La Mémoire des forêts. Actes du colloque « Forêt, archéologie et environnement » 14 au 16 décembre 2004, Nancy, ONF/INRA/DRAC Loraine, 2007.

Fraysse, J. et C., " Enigmatiques cercles de pierres recouverts par un tumulus », Bulletin de la Société d'études scientifiques de l'Anjou, t. IV, 1961, p. 21-29.

Galinié H., Ville, Espace urbain et Archéologie, Tours, Maison des sciences de la ville, de l'urbanisme et des paysages, université François Rabelais, coll. « Sciences de la Ville ${ }^{\circ} 16$ », 2000, URL : https://hal.archives-ouvertes.fr/hal-00537090.

Georges-Leroy, M., Bock, J., Dambrine, É., Dupouey, J.-L., « Le massif forestier, objet pertinent pour la recherche archéologique. L'exemple du massif forestier de Haye (Meurthe-et-Moselle) », Revue géographique de l'Est, vol. 49, n² 2-3, janvier 2009, URL : http://rge.revues.org/1931

Jacquet, G., « La forêt en Val de Loire aux périodes pré-industrielles : histoire, morphologie, archéologie et dendrologie », thèse de doctorat, université de Tours, 2003.

Kooistra, M. J., Maas, G. « The Widespread Occurrence of Celtic Field Systems in the Central Part of the Netherlands ", Journal of Archaeological Science, 35 (8), 2008, p. 2318-2328, doi : 10.1016/j.jas. 2008.03.007.

Kraus, K., Pfeifer, N. « Determination of Terrain Models in Wooded Areas with Airborne Laser Scanner Data ", ISPRS Journal of Photogrammetry and Remote Sensing, 53 (4), p. 193-203, doi : 10.1016/S0924-2716(98)00009-4.

Laplaige, C., « Comparaison de signaux (géophysique, LiDAR) utilisés dans l'étude des dynamiques anthropiques et naturelles ", thèse de doctorat, université de Franche-Comté, 2012, URL : https:// tel.archives-ouvertes.fr/tel-00732379 
Laplaige, C., Rodier, X., Magiorani, L., Crozet, A., « SOLiDAR : diachronie de l'occupation du sol : télédétection en forêt de Chambord, Boulogne, Russy, Blois ", rapport d'activité 2015, SRA Centre-Val de Loire, 2015, $112 \mathrm{p}$.

Magiorani, L., Document final de synthèse, 1999 (SRA Centre Val de Loire).

Magiorani, L., Document final de synthèse, 2000 (SRA Centre Val de Loire).

Magiorani, L., Document final de synthèse, 2001 (SRA Centre Val de Loire).

Magiorani, L., Document final de synthèse, 2002 (SRA Centre Val de Loire).

Magiorani, L., Document final de synthèse, 2003 (SRA Centre Val de Loire).

Magiorani, L., Document final de synthèse, 2004 (SRA Centre Val de Loire).

Magiorani, L., Document final de synthèse, 2005 (SRA Centre Val de Loire).

Magiorani, L., Document final de synthèse, 2006 (SRA Centre Val de Loire).

Magiorani, L., Document final de synthèse, 2007 (SRA Centre Val de Loire).

Magiorani, L., Document final de synthèse, 2008 (SRA Centre Val de Loire).

Magiorani, L., Document final de synthèse, 2009 (SRA Centre Val de Loire).

Magiorani, L., Document final de synthèse, 2010 (SRA Centre Val de Loire).

Magiorani, L., Document final de synthèse, 2011, Prospections en forêt de Russy (SRA Centre Val de Loire).

Martin-Demézil, J. « Les forêts du comté de Blois ", Mémoires de la Société des sciences et lettres de Loir-et-Cher, Blois, 1963, vol. 34 : p. 127-236.

Martin-Demézil, J. « Les forêts du comté de Blois », Mémoires de la Société des Sciences et Lettres de Loir-et-Cher, Blois, 1964, vol. 35, p. 117-188.

Nuninger, L., Oštir, K., Kokalj, Z., Georges-Leroy, M., Opitz, R. « La Télédétection », dans Bertoncello, F. et Rodier, X. (dir.), Information spatiale et Archéologie, coll. « Archéologiques », Paris, Errance, 2011, URL : https://halshs.archives-ouvertes.fr/halshs-00617316.

Opitz, R., Cowley, D., Interpreting Archaeological Topography : Lasers, 3D Data, Observation, Visualisation and Applications, Oxford, Oxbow Books, 2013, p. 288.

Risbøl, O., Bollandsås, O., Nesbakken, A., Ørka, H., Næsset, E., Gobakken, T., « Interpreting Cultural Remains in Airborne Laser Scanning Generated Digital Terrain Models : Effects of Size and Shape on Detection Success Rates ", Journal of Archaeological Science, 40 (12), 2013, p. 4688-4700, doi : 10.1016/j.jas.2013.07.002.

Serres, O. de., Le Théâtre d'agriculture et mesnage des champs, Arles, Actes Sud, 2001.

Schneider, A., Takla, M., Nicolay, M., Raab, A., Raab, T. « A Template-Matching Approach Combining Morphometric Variables for Automated Mapping of Charcoal Kiln Sites », Archaeological Prospection, $\mathrm{n}^{\circ} 22$ (1), 2015, p. 45-62, doi : 10.1002/arp.1497.

Sittler, B., Hauger, K., « Les apports du Laser aéroporté à La documentation de parcellaires anciens fossilisés par la forêt : l'exemple des champs bombés de Rastatt en Pays de Bade ", dans Dupouey, J.-L., Dambrine, E., Dardignac, C., Georges-Leroy, M. (dir.), La Mémoire des forêts. Actes du colloque " Forêt, archéologie et environnement » 14 au 16 décembre 2004, Nancy, ONF/INRA/DRAC Loraine, 2007, p. 155-161. 
Zadora-Rio, E., «Parcs à gibier et garennes à lapins : contribution à une étude archéologique des territoires de chasse dans le paysage médiéval », Hommes et Terres du Nord. Du pollen au cadastre, 2(3), 1986, p. 133-139, URL : https://halshs.archives-ouvertes.fr/halshs-01154800

\section{NOTES}

1. SOLiDAR a débuté en décembre 2014 et s'inscrit dans la dynamique Intelligence des Patrimoines de l'Université François-Rabelais de Tours. Il est piloté par l'UMR 7324 Citeres-LAT et financé par la région Centre, le Domaine national de Chambord et la DRAC Centre. Le programme regroupe des chercheurs de l'UMR 7324 Citeres-LAT, du GéHCO (EA 6293), de l'USR 3124 MSHE C.N. Ledoux, de l'Irstea (Institut national de recherche en sciences et technologies pour l'environnement et l'agriculture), du Domaine national de Chambord, de la DRAC Centre et de l'ONF.

2. Champs bombés : «formes du modelé agraire traditionnel ayant généré une succession de crêtes et de creux» (Sittler et Hauger, 2007, p. 155).

3. Les anomalies phytographiques correspondent à des différences de hauteur et de couleur dans la pousse des cultures, dues à une modification des propriétés du sol à cause de la présence éventuelle de vestiges archéologiques.

4. Chablis : terme utilisé en archéologie et en foresterie pour désigner le creux dans le sol laissé par un arbre déraciné, et par extension la motte formée par les racines de cet arbre.

\section{RÉSUMÉS}

La forêt est un milieu propice à la conservation des vestiges anthropiques, car l'action de l'érosion y est moins importante que dans les milieux ouverts. L'archéologie dans ce milieu porte, d'une part, sur les vestiges de toutes natures conservés sous le couvert forestier et, d'autre part, sur les activités humaines liées à l'exploitation de la forêt (Dupouey et al., 2007). Ces deux approches ont été mises en œuvre dans le cadre du programme SOLiDAR, notamment grâce à une campagne de télédétection LiDAR menée durant l'hiver 2015, dont nous présentons ici les premiers résultats. La zone étudiée comprend le domaine national de Chambord et les forêts domaniales de Boulogne, Russy et Blois, soit un espace forestier de près de 25000 ha. En complément des campagnes de prospection pédestres menées pendant 15 ans, le relevé LiDAR a permis la reconnaissance de vestiges invisibles à l'œil nu touchant à une exploitation ancienne de la forêt et à des occupations humaines antérieures à la forêt. Cette approche diachronique, conjuguant les sources habituellement utilisées par les archéologues (fouilles et prospections archéologiques, archives, études géomorphologiques et écologiques) et la technologie LiDAR, permet une démarche régressive pour l'étude du paysage forestier dans le temps long.

Forests are environments favourable to the conservation of anthropogenic vestiges because the action of erosion is less marked in forests than in open areas. The archaeological work conducted in these forests focuses on all kinds of vestiges preserved by the cover of vegetation as well as human forestry activities (Dupouey et al., 2007). These two approaches were used within the framework of the SOLiDAR programme, namely thanks to the LiDAR remote detection survey conducted during the winter of 2015 , the first findings of which are presented here. The area 
studied includes the Chambord national estate and the state-owned forests of Boulogne, Russy and Blois, representing a woodland area of close to 25000 hectares. In addition to pedestrian surveys conducted over a period of 15 years, the LiDAR survey made it possible to identify vestiges which are invisible to the naked eye and which concern the former exploitation of the forest as well as prior human settlements. This diachronic approach, combining sources widely used by archaeologists (archaeological digs and excavations, archives, geomorphological and ecological surveys) and LiDAR technology makes it possible to use regressive analysis in the longterm study of forestry landscapes.

\section{INDEX}

Keywords : archaeology of the landscape, forest, LiDAR, archaeological vestiges

Mots-clés : archéologie du paysage, forêt, LiDAR, vestiges archéologiques

\section{AUTEURS}

\section{AUDE CROZET}

Aude Crozet est doctorante en archéologie au laboratoire Archéologie et Territoire, UMR 7324 Citeres, université François-Rabelais de Tours/CNRS. Son sujet de thèse porte sur les «Dynamique de peuplement, valorisation des patrimoines et gestion forestière dans le domaine national de Chambord et les forêts attenantes (41)» aude.crozet[at]univ-tours[dot]fr http://citeres.univ-tours.fr/spip.php?article2270

\section{CLÉMENT LAPLAIGE}

Clément Laplaige est ingénieur de recherche au laboratoire Archéologie et Territoire, UMR 7324 Citeres, université François-Rabelais de Tours/CNRS, coresponsable du programme SOLiDAR. clement.laplaige[at]univ-tours[dot]fr http://citeres.univ-tours.fr/spip.php?article2208

\section{XAVIER RODIER}

Xavier Rodier est ingénieur de recherche HDR au CNRS, directeur adjoint de l'UMR 7324 Citeres, responsable du laboratoire Archéologie et Territoire.

xavier.rodier[at]univ-tours[dot]fr

http://citeres.univ-tours.fr/spip.php?article96). 